\title{
Comparing One and Two Class Classification Methods for Multiple Fault Detection on an Induction Motor
}

\author{
Edward Smart \\ Institute of Industrial Research \\ University of Portsmouth \\ Portsmouth, UK \\ Email: edward.smart@port.ac.uk
}

\author{
David Brown \\ Institute of Industrial Research \\ University of Portsmouth \\ Portsmouth, UK \\ Email: david.j.brown@port.ac.uk
}

\author{
Luke Axel-Berg \\ Stork Bottling and Blowmoulding Machinery Ltd \\ Uxbridge, UK \\ Email: luke.axel-berg@sfds.eu
}

\begin{abstract}
This paper shows that one class classification methods combined with wavelets are capable of detecting the majority of faults on a 3 phase induction motor learning only from healthy data. It has important applications for condition monitoring of electro-mechanical machines in industry as it means that rare and expensive-to-obtain faulty data is not required. The experiments were carried out under laboratory conditions on a small, well worn, 3 phase induction motor, which had bearing faults, imbalance faults, broken rotor bar faults and winding faults imposed on it. A two class support vector machine (SVM) was trained on equal amounts of healthy and faulty data to demonstrate that it has high accuracy when faulty data is readily available. The combination of the one-class SVM and wavelets to the best of the author's knowledge has not been previously attempted but shows acceptable results.
\end{abstract}

\section{INTRODUCTION}

Fault detection and identification is a well researched topic but there are considerable challenges in adapting methods and results from lab based experiments to real world industrial applications such as noisy environments, harsh environmental conditions and the complexity of machinery. In the dairy industry, billions of litres of milk are bottled and capped on machines worth in excess of 1 million GBP, ready for transportation to a supermarket. Breakdowns are very serious as not only does this mean the loss of the production and the need for repairs, but also 8 hours of resterilisation must take place which further increases downtime. Dairy engineers have conservatively calculated losses of at least 50,000 GBP per day of machine downtime [1] in Europe. This fact alone highlights the need for an effective condition monitoring system. There are several desirable properties that such a system should have. The machines are often operated in hostile conditions and the chemicals used for sterilisation can damage sensors. Therefore the system should use a minimal amount of sensors. Vibration sensors would be exposed to these harsh conditions and so current was selected because it can be read from the inverter which is protected from the harsh environment.

When collecting data from industrial machines, the quantity of healthy data is usually far greater than the quantity of faulty data. This imbalance makes it difficult to apply traditional two class classification methods successfully because it becomes hard to determine the boundary between the two classes when one class has such limited data [2]. Furthermore, short
TABLE I. DAIRY INDUSTRY CONDITION MONITORING SCENARIO DETAILS

\begin{tabular}{ll}
\hline Conditions & Values \\
\hline Data Sample Duration & circa 30 seconds \\
Sample Rate & $5 \mathrm{kHz}$ \\
Motor Size & $1.5 \mathrm{~kW}$ \\
Motor Speeds & $700 \mathrm{RPM}, 1000 \mathrm{RPM}$ and 1500RPM \\
Motor Loads & $0 \mathrm{~kW}$ (no load), 0.75kW (half load) and \\
& $1.5 \mathrm{~kW}$ (full load)
\end{tabular}

of damaging the machine in every conceivable way, it is almost impossible to collect data for every fault state. One class classification methods are designed to accurately learn from just healthy data so that any deviations are regarded as abnormal. For condition monitoring of industrial machines they are ideal in principle and this paper will compare one and two class support vector machines (SVM) to identify if the one class SVM can produce an acceptable level of detection and if so, how they compare to the two class SVM.

The dairy filler machines are powered using inverter driven $1.5 \mathrm{~kW} 3$ phase induction motors. The machines themselves have embedded systems on which data collection and analysis can be performed. The rough details of this food industry condition monitoring scenario can be found in table I. The laboratory experiment was conducted under these conditions. Details of the faults imposed on the induction motor can be found in section III.

\section{INDUCTION MOTOR ANALYSIS}

The three phase induction motor is one of the most popular motors used in industry. Its popularity results from its ruggedness and reliability. However, the non-linear nature of its operation makes fault detection a significant challenge. Induction motor fault detection is a well researched area with several advanced signal processing techniques proposed [3], [4]. This paper will consider motor current signal analysis (MCSA) as it is particularly applicable to process machinery. In some industries such as food and packaging, motors and machines operate in hostile environments which can damage sensors. Therefore they prefer the smallest number of sensors possible to accurately diagnosis the condition of the machine. MCSA is a good solution as the current signal can be extracted 
from the inverter which is in a sealed cabinet and protected from hostile conditions.

MCSA has been successfully applied for the detection of the four faults imposed on the motor in this paper. In terms of bearing faults, Blodt et al. [5] undertook a comprehensive review of the stator current model and determined that bearing faults not only induce modulations in the current through torque variations but also through imbalance, leading to extra frequencies to study. For eccentricity type faults, Supengat et al. [6] did several experiments over several different motor loads using vibration and current information. After performing analysis on the signals, they identified significant frequencies which provided fault information as the loading on the motor increased. However, to achieve this information, the motor had five sensors attached to it which might not be ideal in certain industries, such as the food industry where machine cleaning can damage sensors. Broken rotor bars have received much attention. Kim et al. [7] studies variances in impedance due to broken rotor bars to try to detect such a fault. The study shows that it is possible to clearly identify broken rotor bars from the current signal. Benbouzid and Kliman [3] present a thorough review of MCSA techniques with which to analyse broken rotor bar faults. Thomson et al. [8] look at trying to detect winding faults using MCSA. It highlighted through spectral analysis that with at least $20 \%$ of the turns shorted, clear spikes at predicted frequencies could be seen. Siddique et al. [9] provides a review of techniques with which to analyse stator winding faults. Gandhi et al. [10] highlights that wavelet analysis techniques are some of the most efficient for extracting relevant fault features.

\section{INDUCTION MOTOR FAULTS}

Bearing faults and shorted turns in the stator windings are the most common reasons for induction motor failure. This is likely because the main mechanical force passes through the bearings and also brineling can occur from the currents that are passed through it. Stator windings have the most electrical force passing through them.

\section{A. Bearing Faults}

The four types of bearing fault are the inner race, the outer race, the ball bearing and the cage defects. These faults can cause machine vibration at pre-defined frequencies depending on the bearing structure and the shaft rotational speed. This vibration results in air gap eccentricity which will cause variations in flux density. Cage defects, ball bearing defects, inner and outer race defects will produce frequencies which can be computed from equations which depend on parameters such as the motor shaft rotational frequency in hertz, the diameter of a ball bearing, the diameter of the bearing itself, the contact angle between the ball bearings and the cage and the number of balls. A full description of these equations and their derivation can be found here [11].

These frequencies result in harmonics in the stator current which can be detected via the following equation:

$$
f_{\text {current }}=\left|f_{s} \pm m f_{v b r}\right|
$$

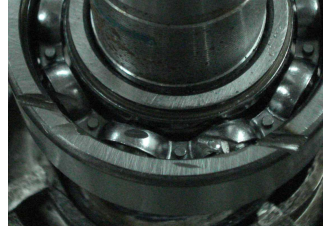

(a) Damaged Bearing

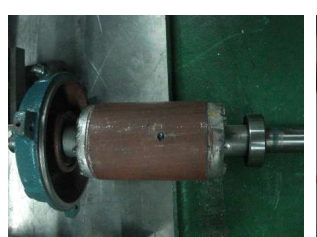

(c) Rotor Bar Damage

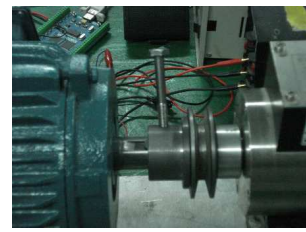

(b) Bolt generating imbalance

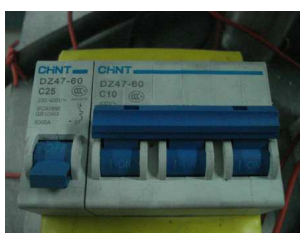

(d) Winding fault through phase disconnection
Fig. 1. Pictures of the four simulated faults on the 3 phase induction motor

where $f_{\text {current }}$ are the current harmonics, $f_{s}$ is the supply frequency, $m$ is a positive integer and $f_{v b r} \in$ $\left\{f_{c d}, f_{b d}, f_{i r}, f_{\text {or }}\right\}$ are the characteristic vibration frequencies for each of the four bearing faults (cage defects, ball bearing defects, inner and outer race defects respectively.

Subfigure (a) in Figure 1 shows the damaged bearing used in this paper. A slot was cut across the bearing, creating all four faults. The authors chose to damage the bearing in this way so that they could compare the performance of fast fourier transforms (FFT) and wavelet packet transforms (WPT) over all four bearing faults. The outer race has been damaged in two places and clear damage can be seen to the cage and one of the balls. Inner race damage (thick grey line) can be seen in the middle of the image.

\section{B. Eccentricity Faults}

Air gap eccentricity occurs in induction motors when there is an unequal gap between the stator and rotor. There are two different types of eccentricity: static and dynamic. Static eccentricity occurs when the axis of rotation is displaced such that the minimal airgap length is constant. This can be caused by the incorrect positioning of the rotor in the stator at the time of manufacture or perhaps through stator ovality. This means that the field distribution in the airgap is no longer symmetrical which leads to a radial force, known as the unbalanced magnetic pull, which acts in the direction of the minimal airgap. Dynamic eccentricity occurs when the rotor is not rotating on its own axis and so the minimal airgap length varies according to the rotated position.

In real world conditions, static and dynamic eccentricity tend to co-exist which gives rise to frequency components in the current spectrum [6].

In this paper, this eccentricity fault is simulated by attaching a bolt to the rotor shaft to create an imbalance (see subfigure (b) in figure 1).

\section{Broken Rotor Bar Faults}

If there is any asymmetry in the supply or the stator winding impedances then a backward rotating field will appear. 
Applying this to the rotor winding means that the frequency of the induced electromagnetic force will appear at slip frequency rather than the supply frequency. Therefore the frequencies for which signs of broken rotor bar faults appear in the stator current can be computed [12].

Subfigure (c) in Figure 1 shows how the rotor bar fault was created by the drilling of a small hole through one of the rotor bars to disconnect.

\section{Stator Winding Faults}

For low voltage stator windings, there are several possible faults that can occur:

- Turn to turn shorts within the coil

- Shorting between coils of the same phase

- Phase to phase shorting

- $\quad$ Phase to earth shorting

Often there is no warning and there is little that online monitoring can do to provide any timely indication of a fault. However, for shorted turns within the stator winding, it is possible to detect their frequency components $\left(f_{w}\right)[8]$.;

Subfigure (d) in figure 1 shows how the winding fault was replicated via the disconnection of one of the phases.

\section{WAVELETS}

Wavelets [13] are a powerful tool for analysing stationary and non-stationary transient signals. They feature the dilation property which allows them to adjust the width of the frequency band and the location of its central frequency so they can automatically focus on the positions of high and low frequency changes. Gaeid et al. [14] provides a good review of wavelets and their useful applicability to induction motor fault detection.

For any signal $x(t) \in L^{2}(\mathbb{R})$ where $t$ is time, the continuous wavelet transform is given by the convolution of the signal with a scaled conjugated wavelet $W(\alpha, \beta)$ where * denotes the complex conjugate of the wavelet $\psi$; namely

$$
W(\alpha, \beta)=\alpha^{-1 / 2} \int_{-\infty}^{\infty} x(t) \psi^{*}\left(\frac{t-\beta}{\alpha}\right) d t .
$$

The term $W(\alpha, \beta)$ indicates how similar the wavelet and signal are through the scale (or pseudo frequency) parameter $\alpha$ and time shift parameter $\beta$. It shows that wavelets are a time-frequency analysis tool. To chose the scale and time shift parameters, it is noted that only dyadic scales can be used without information loss, leading to the discrete wavelet transform, given by:

$$
\psi_{m, n}(t)=2^{-m / 2} \psi\left(2^{-m} t-n\right)
$$

where $\alpha=2^{m}$ and $\beta=n 2^{m}$. These discrete wavelets also form an orthonormal basis. Wavelet analysis can then be performed via a low-pass filter $h(n)$ relating to the scaling function $\varphi(t)$ and a high-pass wavelet filter $g(n)$ that is related to the wavelet function $\psi(t)$ :

$$
\begin{aligned}
& h(n)=2^{-1 / 2}\langle\varphi(t), \varphi(2 t-n)\rangle \\
& g(n)=2^{-1 / 2}\langle\phi(t), \phi(2 t-n)\rangle .
\end{aligned}
$$

In decomposition of the signal $x(t)$, the application of the low and high pass filters leads to two vectors $c A_{1}$ (approximation coefficients) and $c D_{1}$ (detail coefficients). In wavelet transform decomposition, this step is repeated on the approximation vector to achieve the required depth of decomposition. The symbol $\downarrow 2$ denotes down-sampling (omitting the odd indexed elements of the filter).

WPT [13] are a generalisation of the wavelet transform. Define two functions $W_{0}(t)=\varphi(t)$ and $W_{1}(t)=\psi(t)$ where $\varphi(t)$ is the scaling function and $\psi(t)$ is the wavelet function. In the orthogonal case, for $m=0,1,2, \ldots$, , functions $W_{m}(t)$ are obtained by:

$$
\begin{gathered}
W_{2 m}(t)=2 \sum_{n=0}^{2 N-1} h(n) W_{m}(2 t-n) \\
W_{2 m+1}(t)=2 \sum_{n=0}^{2 N-1} g(n) W_{m}(2 t-n) \\
W_{j, m, n}(t)=2^{-j} / 2 W_{m}\left(2^{-j} t-n\right) .
\end{gathered}
$$

where $j$ is a scale parameter and $n$ is a time localisation parameter. The functions $W_{j, m, n}$ are called wavelet packet atoms.

The difference between this method and wavelet transforms is that both the details and the approximations are further decomposed, thus giving a wavelet packet tree. Each decomposition contains a set of nodes, indexed by positive integers $(i, j)$ where $j$ is the node depth and $i$ is the node position at that depth for $i=0,1, \ldots, 2^{j}-1$.

The signal energy for sub-signal node

$$
B_{i}^{j}\left(i=0,1, \ldots, 2^{j}-1\right)
$$

(i.e. the approximation and detail) at depth $j$ is given by:

$$
E_{i}=\frac{100 \sum_{k=1}^{M}\left|B_{i}^{j}(k)\right|^{2}}{\sum_{i=0}^{2^{j}-1}\left(\sum_{k=1}^{M}\left|B_{i}^{j}(k)\right|^{2}\right)}
$$

where the numerator is the energy for a given node, the denominator is the energy of the whole signal and $M$ is the number of sampling points. 


\section{Classifiers}

One class classifiers [15], [16] were chosen because they are designed for novelty detection; in this case the aim is to separate a small number of abnormal data points from a large number of normal data points. They are designed to for situations where one class (the healthy class) is well sampled and the other class (the faulty class) is very poorly sampled, making it hard to use information from the poorly sampled class to determine a boundary between the two classes.

\section{A. One Class Classification - Support Vector Novelty Detec- tion}

One Class Support Vector Machine (OCSVM) [17] is a novelty detection method based on the support vector machine [18].

Consider healthy training data $x_{1}, x_{2}, \ldots, x_{l} \in \mathbb{R}^{n}$. Let $\phi$ be the mapping $\phi: \mathbb{R} \rightarrow F$ into some feature dot product space $F$. Let $k(x, y)=(\phi(x), \phi(y))$ be a positive definite kernel which operates on the mapping $\phi$. In this paper, the kernel used is the Gaussian kernel, $k(x, y)=\exp \left(-\|x-y\|^{2} / 2 \sigma^{2}\right)$, as it suppresses growing distances in larger feature spaces. Here, $\sigma$ is the width parameter associated with the Gaussian kernel. The data is mapped into the feature space via the kernel function and is separated from the origin with maximum margin. The decision function is found by minimising the weighted sum of the support vector regulariser and the empirical error term depending on a margin variable $\rho$ and individual error terms $\xi_{i}$ :

$$
\begin{array}{cl}
\min _{w \in F, \xi \in \mathbb{R}^{l}, \rho \in \mathbb{R}} & \frac{1}{2}\|w\|^{2}+\frac{1}{\nu l} \sum_{i=1}^{l} \xi_{i}-\rho, \\
\text { subject to } & \left(w \cdot \phi\left(x_{i}\right)\right) \geq \rho-\xi_{i} \\
& \xi_{i} \geq 0
\end{array}
$$

where $w$ is a weight vector in $F$ and $v$ is the fraction of the training set to be regarded as outliers. Using Lagrangian multipliers, $\alpha_{i}, \beta_{i} \geq 0$, with constraints and setting the derivatives of those multipliers with respect to $w$ equal to zero leads to

$$
\begin{aligned}
w & =\sum_{i=1}^{l} \alpha_{i} \phi\left(x_{i}\right), \\
\sum_{i=1}^{l} \alpha_{i} & =1, \\
\alpha_{i}+\beta_{i} & =\frac{1}{\nu l} .
\end{aligned}
$$

Solving the dual problem leads to the 'abnormality' detection function, given by

$$
g(x)=\rho_{0}-\sum_{i=1}^{N_{s}} \alpha_{i} k\left(s_{i}, x\right) .
$$

The user has to choose the appropriate kernel, with its associated parameters, for the problem. Rather than choosing an error penalty $C$ as via the classical SVM method, one chooses a value for $\nu$ which is the fraction of the training set to be classified as outliers. The software used for this classifier is LIBSVM for Matlab version 3.12 [19].
Fig. 2. Photo of the motor and data acquisition unit

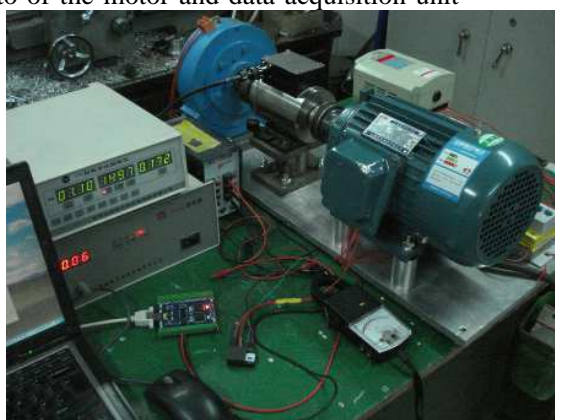

\section{B. Two Class Classification - Support Vector Classification}

The two class SVM classifier [18] seeks to maximise the margin between the healthy and faulty classes but classification performance can suffer when one of the classes is undersampled. Using the same variables in section V-A, the optimisation problem becomes

$$
\begin{aligned}
& \min _{w \in F, \xi \in R^{l}, \rho \in R} \frac{1}{2}\|w\|^{2}-\nu \rho+\frac{1}{l} \sum_{i=1}^{l} \xi_{i}, \\
& \text { subject to } y_{i} \cdot\left(\left(x_{i} \cdot w\right)+b\right) \geqslant \rho-\xi_{i}, \quad \xi_{i} \geqslant 0, \rho \geqslant 0
\end{aligned}
$$

with decision function

$$
g(x)=\operatorname{sgn}\left(\sum_{i=1}^{l} y_{i} \alpha_{i} K\left(x_{i}, x\right)+b\right)
$$

\section{DATA ACQUISITION}

The data was acquired from a 3 phase asynchronous $380 \mathrm{~V} 1.5 \mathrm{~kW}$ induction motor. Motor speed was altered by using a Vacon 10 Amp inverter drive. Current was read from an open loop sensor in the inverter. Five motor states are considered; healthy, faulty bearing, eccentricity, broken rotor bar and stator winding. Three motor speeds are considered; 700RPM, 1000RPM and 1500RPM. Three different loads are also considered; 0kW (no load), $0.75 \mathrm{~kW}$ (half load) and $1.5 \mathrm{~kW}$ (full load). Only one phase of current was used and three experimental runs were made for each motor configuration. For each combination of these states, three sets of roughly 30 seconds of current signal was collected, except in the case of the winding fault whereby only data from the machine running at 1500RPM was collected. This was because it was impossible to simulate the winding fault by disconnecting a phase at 700 RPM and 1000 RPM as the inverter was needed to achieve these speeds. This means that there were a total of $(5 * 3 * 3 * 3)$ $(2 * 3 * 3)=117$ sets of data collected. The experimental layout can be found in figure 2 .

\section{METHOD}

\section{A. Method Details}

In the section, the experimental design is introduced so that the one class and two class SVMs could be compared. 
TABLE II. PARAMETER RANGES FOR BOTH CLASSIFIERS

\begin{tabular}{ll}
\hline Parameter & Values \\
\hline$\sigma$ & $10^{i}$ for $i=-9,-8, \ldots,-1,0,1,2$ \\
$\nu$ & $0.01,0.05,0.1,0.15,0.2,0.3,0.4,0.5$
\end{tabular}

1) The 117 signal samples were analysed using a Hanning window of length 16000 (found to be effective from initial experimentation).

2) Each signal was sampled at $5 \mathrm{kHz}$ and processed using WPT with the daubechies 10 wavelet to depths varying from 3 and 11 inclusive in unit intervals. The signal energy was computed for each node. Db10 was chosen as it had previous success in fault detection [20].

3) Data samples were split into three different frequency feature sets of signals energies in the following ranges: $0-125 \mathrm{~Hz}, 0-500 \mathrm{~Hz}$ and $0-2500 \mathrm{~Hz}$.

4) The training set for one class classification was formed by combining two healthy signal samples from each load, each speed and each frequency feature set so that the third remaining sample for each healthy load, speed and feature set forms a healthy testing set. This means that for the training set (healthy data only) for the 700 RPM data on the $0-125 \mathrm{~Hz}$ feature set, for example, consists of 6 signal samples ( 2 lots for each of the 3 loads). The testing set has the 3 remaining healthy samples (one for each load) and the faulty data (bearing, shaft imbalance, broken rotor bar or stator winding). For two class classification, the training set was formed by combining two healthy and two faulty (bearing or shaft imbalance or rotor or winding fault) signal samples from each load, speed and frequency feature set so that the third remaining sample for each healthy and faulty load, speed and feature set forms a healthy testing set. This means that for the training set for the 700 RPM data on the $0-125 \mathrm{~Hz}$ feature set, for example, consists of 12 signal samples (2 lots for each of the 3 loads from 2 states (healthy and faulty)). The testing set has the 3 remaining healthy samples (one for each load) and the 3 remaining faulty data samples (bearing, shaft imbalance, broken rotor bar or stator winding).

5) Each of the classifiers were trained using all parameters in the given range (see table II). 10 fold cross validation was applied during training.

6) The classifiers were then tested using the testing sets for each fault, speed and frequency feature set.

For extra clarity, although the training sets contain current data with variable loads (for a given speed), data from different speeds were not compared against each other as this would lead to results bias.

\section{PERFORMANCE METRICS}

For the traditional two class classification problem where data from each class is roughly equal, there are many metrics such as accuracy, sensitivity and specificity which can be used to measure performance. For one class classification problems, where the data set is heavily imbalanced to favour the healthy class, accuracy, for example, is not as useful. Consider a test set with 90 healthy and 10 unhealthy data points. A classifier that classifies all points as healthy will have an accuracy of $90 \%$ but is likely to be very poor for detecting unhealthy data.

The balanced error rate (BER) is given as the average of the false positive and false negatives

$$
B E R=1 / 2(F P+F N)
$$

where $F P$ and $F N$ denotes the percentage of wrongly classified healthy and unhealthy data respectively. In this example, the BER would be 50\%; demonstrating how weak the given classifier is. This metric will be used throughout the results section to judge performance.

\section{RESUlTS}

In this section the results are presented for the one class and two class SVMs so that the BER can be compared for each motor speed and feature set. The two class SVM was trained on BOTH healthy and faulty data which was plentiful and roughly equal in quantity so its performance will be superior to that of the one class SVM which was only trained on healthy data. The two class SVM demonstrated very low errors on the bearing faults (bar 1500RPM), the imbalance faults, the rotor bar faults and the winding faults. This shows that given sufficient faulty data, automatic fault detection can be achieved with a strong level of accuracy (see tables III to VI) using a single phase of current data rather than multiple vibration sensors.

In many industries however, faulty data is difficult or impossible to obtain without incurring significant cost. Even if some data is available, it is highly unlikely it will encompass all fault conditions. One class classification methods focus on healthy data, which is readily available and learn only from this data. Tables III to VI show the performance of the one class SVM and on internal motor faults (bearing (bar 1500RPM), rotor bar and winding), the average classification error is less than $10 \%$ which is a very good performance, given it was required to detect unknown faults. Its performance on bearing faults at 1500RPM was little better than chance which is a problem. However, this fault at this speed produced the weakest performance from the two class SVM with average errors of nearly $22 \%$ on healthy and faulty test data. It is felt that the weak performance on the 1500RPM bearing data was due to the motor compensating for the fault as it was designed to run at that speed.

The one class SVM also performed reasonably well on the imbalance fault with a lowest average classification error of $18.4 \%$. This fault is a very difficult one to detect given it is external to the motor, the metal bolt is not the biggest and also the end of the shaft is held by the load generator so any imbalance effects will be minimised.

\section{Conclusion}

The results show that the one class SVM can be effectively used for classification on induction motor fault data using healthy data only. This result has considerable potential for industry as it shows that the majority of faults at different motor speeds can be detected learning from just healthy data. This means that one class classification algorithms can 
TABLE III. THE LOWEST BER RATE USING ONE CLASS AND TWO CLASS SVM OVER ALL SPEEDS AND MOTOR LOADS FOR THE BEARING FAULT

\begin{tabular}{lllll}
\hline Speed/Feat. & $\begin{array}{l}\text { 1SVM } \\
\text { Ave }\end{array}$ & $\begin{array}{l}\text { 1SVM } \\
\text { STD }\end{array}$ & $\begin{array}{l}\text { 2SVM } \\
\text { Ave }\end{array}$ & $\begin{array}{l}\text { 2SVM } \\
\text { STD }\end{array}$ \\
\hline $700 /$ All & $1.9 \%$ & $1.3 \%$ & $0.0 \%$ & $0.0 \%$ \\
$700 / 500$ & $1.5 \%$ & $1.6 \%$ & $0.0 \%$ & $0.0 \%$ \\
$700 / 125$ & $1.8 \%$ & $1.6 \%$ & $0.0 \%$ & $0.0 \%$ \\
$1000 /$ All & $2.0 \%$ & $2.2 \%$ & $0.0 \%$ & $0.0 \%$ \\
$1000 / 500$ & $2.1 \%$ & $1.1 \%$ & $0.0 \%$ & $0.0 \%$ \\
$1000 / 125$ & $1.5 \%$ & $0.6 \%$ & $0.0 \%$ & $0.0 \%$ \\
$1500 /$ All & $48.0 \%$ & $3.8 \%$ & $21.9 \%$ & $0.3 \%$ \\
$1500 / 500$ & $48.3 \%$ & $1.9 \%$ & $21.9 \%$ & $0.3 \%$ \\
$1500 / 125$ & $48.0 \%$ & $1.6 \%$ & $21.9 \%$ & $0.3 \%$
\end{tabular}

TABLE IV. THE LOWEST BER RATE USING ONE CLASS AND TWO CLASS SVM OVER ALL SPEEDS AND MOTOR LOADS FOR THE IMBALANCE FAULT

\begin{tabular}{lllll}
\hline Speed/Feat. & $\begin{array}{l}\text { 1SVM } \\
\text { Ave }\end{array}$ & $\begin{array}{l}\text { 1SVM } \\
\text { STD }\end{array}$ & $\begin{array}{l}\text { 2SVM } \\
\text { Ave }\end{array}$ & $\begin{array}{l}\text { 2SVM } \\
\text { STD }\end{array}$ \\
\hline $700 /$ All & $22.0 \%$ & $3.1 \%$ & $7.5 \%$ & $2.0 \%$ \\
$700 / 500$ & $21.7 \%$ & $1.7 \%$ & $6.9 \%$ & $3.4 \%$ \\
$700 / 125$ & $20.3 \%$ & $2.1 \%$ & $6.9 \%$ & $3.7 \%$ \\
$1000 /$ All & $18.4 \%$ & $1.9 \%$ & $3.6 \%$ & $1.8 \%$ \\
$1000 / 500$ & $19.2 \%$ & $4.7 \%$ & $4.7 \%$ & $1.9 \%$ \\
$1000 / 125$ & $18.9 \%$ & $4.6 \%$ & $4.4 \%$ & $1.9 \%$ \\
$1500 /$ All & $24.0 \%$ & $1.7 \%$ & $2.4 \%$ & $1.5 \%$ \\
$1500 / 500$ & $28.6 \%$ & $2.7 \%$ & $3.5 \%$ & $2.8 \%$ \\
$1500 / 125$ & $24.4 \%$ & $4.0 \%$ & $3.9 \%$ & $2.3 \%$
\end{tabular}

be applied in a wide range of industries which depend on induction motors to power their machines. In principle they would be relatively easy to setup as healthy data is readily available.

\section{ACKNOWLEDGMENT}

Thanks also goes to Stork Blowmoulding and Bottling Machinery for their advice and information on condition monitoring scenarios and issues in the dairy industry.

\section{REFERENCES}

[1] H. Wasif, A. Aboutalebi, D. Brown, and L. Axel-Berg, "Condition monitoring system for process industries a business approach," in Industrial Electronics and Applications (ISIEA), 2012 IEEE Symposium on. IEEE, 2012, pp. 251-256.

[2] D. Tax, A. Ypma, and R. Duin, "Support vector data description applied to machine vibration analysis," in Proc. 5th Annual Conference of the Advanced School for Computing and Imaging, 1999, pp. 15-23.

[3] M. Benbouzid and G. Kliman, "What stator current processing-based technique to use for induction motor rotor faults diagnosis?" Energy Conversion, IEEE Transactions on, vol. 18, no. 2, pp. 238-244, 2003.

[4] A. Bellini, F. Filippetti, C. Tassoni, and G. Capolino, "Advances in diagnostic techniques for induction machines," Industrial Electronics, IEEE Transactions on, vol. 55, no. 12, pp. 4109-4126, 2008.

[5] M. Blodt, P. Granjon, B. Raison, and G. Rostaing, "Models for bearing damage detection in induction motors using stator current monitoring," Industrial Electronics, IEEE Transactions on, vol. 55, no. 4, pp. 1813 1822, 2008.

[6] R. Supangat, J. Grieger, N. Ertugrul, W. Soong, D. Gray, and C. Hansen, "Investigation of static eccentricity fault frequencies using multiple sensors in induction motors and effects of loading," in IEEE Industrial Electronics, IECON 2006-32nd Annual Conference on. IEEE, 2006, pp. 958-963.

[7] B. Kim, K. Lee, J. Yang, S. Lee, E. Wiedenbrug, and M. Shah, "Automated detection of rotor faults for inverter-fed induction machines under standstill conditions," in Energy Conversion Congress and Exposition, 2009. ECCE 2009. IEEE. IEEE, 2009, pp. 2277-2284.
TABLE V. THE LOWEST BER RATE FOR THE ONE AND TWO CLASS SVM OVER ALL SPEEDS AND MOTOR LOADS FOR THE BROKEN ROTOR BAR FAULT

\begin{tabular}{lllll}
\hline Speed/Feat. & $\begin{array}{l}\text { 1SVM } \\
\text { Ave }\end{array}$ & $\begin{array}{l}\text { 1SVM } \\
\text { STD }\end{array}$ & $\begin{array}{l}\text { 2SVM } \\
\text { Ave }\end{array}$ & $\begin{array}{l}\text { 2SVM } \\
\text { STD }\end{array}$ \\
\hline $700 /$ All & $2.8 \%$ & $1.4 \%$ & $0.0 \%$ & $0.0 \%$ \\
$700 / 500$ & $1.5 \%$ & $1.6 \%$ & $0.0 \%$ & $0.0 \%$ \\
$700 / 125$ & $1.7 \%$ & $1.3 \%$ & $0.0 \%$ & $0.0 \%$ \\
$1000 /$ All & $6.7 \%$ & $4.4 \%$ & $0.0 \%$ & $0.0 \%$ \\
$1000 / 500$ & $2.2 \%$ & $1.6 \%$ & $0.0 \%$ & $0.0 \%$ \\
$1000 / 125$ & $1.7 \%$ & $2.2 \%$ & $0.0 \%$ & $0.0 \%$ \\
$1500 /$ All & $7.7 \%$ & $2.7 \%$ & $0.0 \%$ & $0.1 \%$ \\
$1500 / 500$ & $6.9 \%$ & $4.2 \%$ & $0.0 \%$ & $0.1 \%$ \\
$1500 / 125$ & $6.0 \%$ & $4.1 \%$ & $0.0 \%$ & $0.1 \%$
\end{tabular}

TABLE VI. THE LOWEST BER RATE FOR THE ONE AND TWO CLASS SVM OVER ALL SPEEDS AND MOTOR LOADS FOR THE STATOR WINDING FAULT

\begin{tabular}{lllll}
\hline Speed/Feat. & $\begin{array}{l}\text { 1SVM } \\
\text { Ave }\end{array}$ & $\begin{array}{l}\text { 1SVM } \\
\text { STD }\end{array}$ & $\begin{array}{l}\text { 2SVM } \\
\text { Ave }\end{array}$ & $\begin{array}{l}\text { 2SVM } \\
\text { STD }\end{array}$ \\
\hline $1500 /$ All & $1.8 \%$ & $1.6 \%$ & $0.0 \%$ & $0.0 \%$ \\
$1500 / 500$ & $1.5 \%$ & $1.6 \%$ & $0.0 \%$ & $0.0 \%$ \\
$1500 / 125$ & $1.7 \%$ & $1.4 \%$ & $0.0 \%$ & $0.0 \%$
\end{tabular}

[8] W. Thomson and M. Fenger, "Current signature analysis to detect induction motor faults," Industry Applications Magazine, IEEE, vol. 7, no. 4, pp. 26-34, 2001

[9] A. Siddique, G. Yadava, and B. Singh, "A review of stator fault monitoring techniques of induction motors," Energy conversion, ieee transactions on, vol. 20, no. 1, pp. 106-114, 2005.

[10] A. Gandhi, T. Corrigan, and L. Parsa, "Recent advances in modeling and online detection of stator inter-turn faults in electrical motors," Industrial Electronics, IEEE Transactions on, vol. 58, no. 5, pp. 1564 $1575,2011$.

[11] B. Li, M. Chow, Y. Tipsuwan, and J. Hung, "Neural-network-based motor rolling bearing fault diagnosis," Industrial Electronics, IEEE Transactions on, vol. 47, no. 5, pp. 1060-1069, 2000.

[12] B. Yazici and G. Kliman, "An adaptive statistical time-frequency method for detection of broken bars and bearing faults in motors using stator current," Industry Applications, IEEE Transactions on, vol. 35, no. 2, pp. 442-452, 1999.

[13] D. Ruch and P. Van Fleet, Wavelet Theory: An Elementary Approach with Applications. Wiley, 2011. [Online]. Available: http://books.google.co.uk/books?id=gidfj5euyeUC

[14] K. Gaeid and H. Ping, "Wavelet fault diagnosis and tolerant of induction motor: A review," International Journal of the Physical Sciences, vol. 6, no. 3, pp. 358-376, 2011.

[15] S. Khan and M. Madden, "A survey of recent trends in one class classification," Artificial Intelligence and Cognitive Science, vol. 6206, pp. 188-197, 2010

[16] D. Tax, "One-class classification," Ph.D. dissertation, Delft University of Technology, http://homepage.tudelft.nl/n9d04/thesis.pdf, June 2001.

[17] B. Schölkopf, R. C. Williamson, A. J. Smola, J. Shawe-Taylor, and J. C. Platt, "Support vector method for novelty detection," in Advances in Neural Information Processing Systems 12, S. A. Solla, T. K. Leen, and K.-R. Müller, Eds. Cambridge, MA: MIT Press, 2000, pp. 582588.

[18] B. E. Boser, I. M. Guyon, and V. N. Vapnik, "A training algorithm for optimal margin classifiers," in COLT '92: Proceedings of the fifth annual workshop on Computational learning theory. New York, NY, USA: ACM, 1992, pp. 144-152.

[19] C.-C. Chang and C.-J. Lin, LIBSVM: a library for support vector machines, 2001, software available at http://www.csie.ntu.edu.tw/ $\sim_{\mathrm{cjlin} /}$ libsvm/.

[20] J. Cusido, L. Romeral, J. A. Ortega, J. A. Rosero, and A. Garcia Espinosa, "Fault detection in induction machines using power spectral density in wavelet decomposition," Industrial Electronics, IEEE Transactions on, vol. 55, no. 2, pp. 633-643, 2008. 